Fanaras Konstantinos' ${ }^{1}$ Reinhard Heun ${ }^{2}$

\title{
The effects of Rhodiola Rosea supplementation on depression, anxiety and mood - A Systematic Review
}

\author{
'Undergraduate student of medicine at Aristotle University of Thessaloniki, Aristotle University of Thessaloniki, Thessaloniki Greece \\ ${ }^{2}$ Professor of Psychiatry and Psychotherapy, University of Bonn, Bonn, Germany \\ *email: kostaskfayahoo.com
}

DOI: 10.2478/gp-2019-0022

Received: 19 October 2019; Accepted: 19 December 2019

\begin{abstract}
Objectives: Rhodiola rosea is an adaptogen herb from the Crassulaceae family, which has been vastly used in the Russian and Chinese medicine. The herb is used against depression, anxiety, mental and physical fatigue and to promote overall health. In this systematic review, we examined the effects of $R$. rosea on depression, anxiety and mood, as these are the most relevant to mental health.

Methods: Literature searches were made in PubMed using the term 'Rhodiola rosea'. Inclusion criteria were: Randomized controlled trials using interventions of $R$. rosea on any type of participants, while focusing on the effects of the intervention on depression, anxiety or mood. Mixed interventions of $R$. rosea with other herbs were excluded. Studies not published in English or Greek were excluded. Results: A total of 39 randomized controlled trials were identified and their abstract was screened. After screening, a total of 17 papers were excluded because they were focusing on irrelevant outcomes. The full text of the remaining 22 papers was read and an additional 17 papers were excluded. These papers were excluded because they were eventually not focusing on our main outcome or they were using $R$. rosea interventions with other herbs. In the end, a total of 5 papers ( $n=327$ participants) were found eligible for our systematic review. In these studies, $R$. rosea seems to improve the symptoms of mild to moderate depression, symptoms of mild anxiety and to enhance mood. The last date of our search was October 13, 2019.

Conclusion: Rhodiola rosea supplementation may alleviate symptoms of mild to moderate depression and mild anxiety, while it may also enhance mood. The findings of our review are not definite due to the lack of available experimental data. Randomized controlled trials with a low risk of bias are needed to further study the herb.
\end{abstract}

\section{Keywords}

Rhodiola rosea, adaptogens, depression, anxiety, mood, systematic review

\section{INTRODUCTION}

Rhodiola rosea is an adaptogen herb used in herbal medicines (Ross, 2014).Adaptogens are naturalhealth products(NHPs) that produce a non-specific response aimed to promote homeostasis (Brekhman, Dardymov, 1969). For a herb to be considered an adaptogen by the European Medicines Agency, it must also be completely safe, non-toxic and to affect various organ systems of the recipient's organism. A common characteristic of adaptogens, is their ability to reduce stress, by interfering in the HPA axis. At stressful events, adaptogens are thought to hinder the release of corticotropic-releasing hormone (CRH), cortisol, nitric oxide, pro-inflammatory cytokines, free radicals, stress-activated protein kinase (SAPK) and Jun N-terminal protein kinase (JNK), while also blocking the stress-induced decrease of BDNF, ATP production, glucocorticoid receptor sensitivity, HPS-70 and neuropeptide-Y (Panossian, Wikman, 2010; Anghelescu et al., 2018). The term adaptogen was first introduced in 1947 (European Medicines Agency 2008) and was vastly used in the Soviet Union (Brekhman, Dardymov, 1969). Most research on adaptogens was made in the Soviet Union, Korea and China. However, flaws in the studies, lack of research and data prevented the worldwide acceptance of adaptogens into clinical practice (European Medicines Agency 2008). Some of the most common adaptogen herbs are Ashwagandha, Panax Ginseng, Schisandra and Rhodiola Rosea (Panossian et al., 2007). Rhodiola rosea is a perennial flowering plant of the family Crassulaceae (Plants for a Future 2012). The plant grows naturally at Arctic regions of Europe, Asia (mostly Siberia), and in the eastern coastal regions of North America (Plants for a Future 2012). It reaches a height of up to forty centimetres and has yellow flowers (Ross, 2014). Approximately 140 
different chemicals were found in the roots of Rhodiola rosea (Panossian, Wikman, Sarris, 2010). Some of these chemicals are rosavin, rosin, rosarin, phenols, organic acids, terpenoids, phenolic acids, flavonoids, alkaloids, tyrosol, anthraquinones and salidroside (Evstavieva et al., 2010), (Mao, Li, Yao, 2007). Extracts of Rhodiola rosea used in human clinical studies are standardized at $>3.0 \%$ rosavins, $>0.8 \%$ salidrosides at a ratio of 3:1 (Ross, 2014). These two substances are thought to be responsible for the anxiolytic and antidepressant actions of the herb (PerfumI, Mattioli, 2007); (Mattioli, Funari, Perfumi, 2008) and are considered the marker compounds of Rhodiola rosea (Ross, 2014).

The history of Rhodiola rosea as a medical herb traces back to $77 \mathrm{AD}$, when the Greek physician Dioscorides first recorded the plants' medical applications in his medical book De Materia Medica (Mell 1938 as cited by Ross 2014). The herb was used by the Vikings to enhance physical strength and endurance, and in Tibetan medicine, to treat lung disorders (Ross, 2014). In the $18^{\text {th }}$ century, the Swedish Botanist Carolus Linnaeus who is recognized as the 'Father of Taxonomy' renamed the herb to Rhodiola rosea for the rose-like aroma of the flowers and used the herb for the treatment of leukorrhea, hysteria and headache (Ross, 2014). In the $19^{\text {th }}$ century, Rhodiola rosea was used in France as a brain tonic and in Germany for headaches, pain relief, haemorrhoids treatment, as a stimulant and as an anti-inflammatory agent (Ross, 2014). In 1969, it had already been approved and registered by the Soviet Ministry of Health for medical use. Since 1985, R. rosea has been recorded in Herbal Medicinal Product in Sweden. R. rosea has now been documented by European Medicines Agency for temporary relief of symptoms of stress.

The pharmacology involved in the antidepressant effects of the herb has been examined by numerous studies. Amsterdam, Panossian (2016) reported that the herb seems to stimulate expression and release of neuropeptide- $Y$ in neuroglial cells, to regulate more than 50 genes involved in the regulation of behaviour, mood and depressive disorders and to be overall associated with the key mediators of stress response, regulation of homeostasis of HPA axis activity, modulation of G-protein coupled receptor (GPCR) signalling pathways and other molecular networks involved in depression. Other research has shown that Rhodiola rosea acts as a MAO-A and MAO-B inhibitor, showing evidence about the herb's antidepressant and cognitive enhancing properties (van Diermen et al 2009). R. rosea also seems to stimulate noradrenalin, serotonin, dopamine and acetylcholine receptors in the brain (Mao et al., 2015). Finally, (Lishmanov et al., 1987) reports that studies have also shown that R. rosea promotes an increase in $\beta$-endorphins, which lowers the intensity of the stress response and the production of endorphins at stressful situations. In other words, the herb acts by decreasing or totally preventing stress-induced hormonal changes, by its adaptogenic properties (Figure 1).

The objective of this systematic review was to examine the effects of R. rosea on humans suffering from depression, anxiety and mood disorders. We hope that our review proves to be informative for the therapeutic effects of the herb and encourages future research.

\section{METHODS}

\section{Type of Study}

The present study is a systematic review of randomized clinical trials on the effects of Rhodiola rosea supplementation on depression, anxiety and mood. Literature searches were made in PubMed. The search term was 'Rhodiola rosea' and the filters focused on randomized controlled trials. Bias was assessed with the Cochrane tool for assessing the risk of bias (Higgins et al., 2011). The study was performed according to the Preferred Reporting Items for Systematic Reviews and Meta-Analysis (PRISMA criteria; Moher D et al., 2009)

\section{Study Selection}

We selected studies using the following inclusion criteria:

a) must use an intervention containing Rhodiola rosea,

b) must examine the effects of the intervention on depression or anxiety or mood,

c) must be a randomized clinical trial,

d) no criteria were set for the participants of the included studies,

e) must be published in English or Greek.

We excluded studies using the following exclusion criteria:

a) The intervention must not be a combination of medicinal herbs. This criterion was set to ensure that the therapeutic effects reported across studies were solely attributed to Rhodiola rosea.

\section{RESULTS}

After using the methods, a total of 39 randomized clinical trials were identified. All of the 39 trial's abstracts were screened. After screening, 17 papers were excluded. These papers were excluded because they were not examining the effects of 
1) Increases heat shock protein HPS-70 (Amsterdam, Panossian, 2016)

$\rightarrow$ Inhibits the stress-induced expression of NO genes

$\rightarrow$ Increases ATP production

$\rightarrow$ Repairs the damage from stress proteins

$\rightarrow$ Inhibits the release of JNK

2) Decreases SAPK/JNK (Amsterdam, Panossian, 2016; Anghelescu et al., 2018)

$\rightarrow$ Decreases the formation of free radicals and NO

$\rightarrow$ Increases ATP production

$\rightarrow$ Decreases corticotropic-releasing hormone (CRH), cortisol secretion and increases glucocorticoid receptor sensitivity

$\rightarrow$ Blocks the cortisol-induced suppression of the immune system

3) Increases neuropeptide-Y (Amsterdam, Panossian, 2016)

$\rightarrow$ Inhibits cortisol secretion

$\rightarrow$ Inhibits pro-inflammatory cytokine IL-1b

$\rightarrow$ Promotes ATP synthesis

4) Increases BDNF (Yang et al., 2014)

$\rightarrow$ Protects neurons from stress and encourages the growth of new neurons

5) Inhibits MAO-A and MAO-B receptors (van Diermen et al., 2009)

$\rightarrow$ Increases dopamine, serotonin, norepinephrine

6) Increases $\beta$-endorphins (Lishmanov et al., 1987)

Decreases the stress response and the stress-induced increase of $\beta$-endorphins

7) Stimulates noradrenalin, serotonin, dopamine and acetylcholine receptors (Mao et al., 2015)

$\rightarrow$ Various anti-depressant, anti-stress and cognitive enhancing effects

Figure 1. 'Mechanism of action of Rhodiola rosea.'

Rhodiola rosea on depression or anxiety or mood. The full text of the remaining 22 papers was read. After full-text reading, 17 more papers were excluded, leaving a final sample of 5 papers. Of these 17 papers, three studies were excluded because they used Rhodiola rosea interventions with other herbs; two studies were excluded because they were assessing mental work; three studies were excluded because they were assessing endurance exercise performance; and the rest of the studies were assessing the effects of Rhodiola rosea on anaerobic exercise performance, physical and mental fatigue, stress-related fatigue, skeletal muscle damage and inflammation, sport performance, skeletal muscle phosphate kinetics, and the effects on oxidantsantioxidants. Finally, two studies were duplicates. The last date of our search was 13 October 2019.

\section{Included Studies}

A total of five randomized controlled clinical trials were found eligible for our review. The population of the studies was participants over the age of 17 years, diagnosed with either 

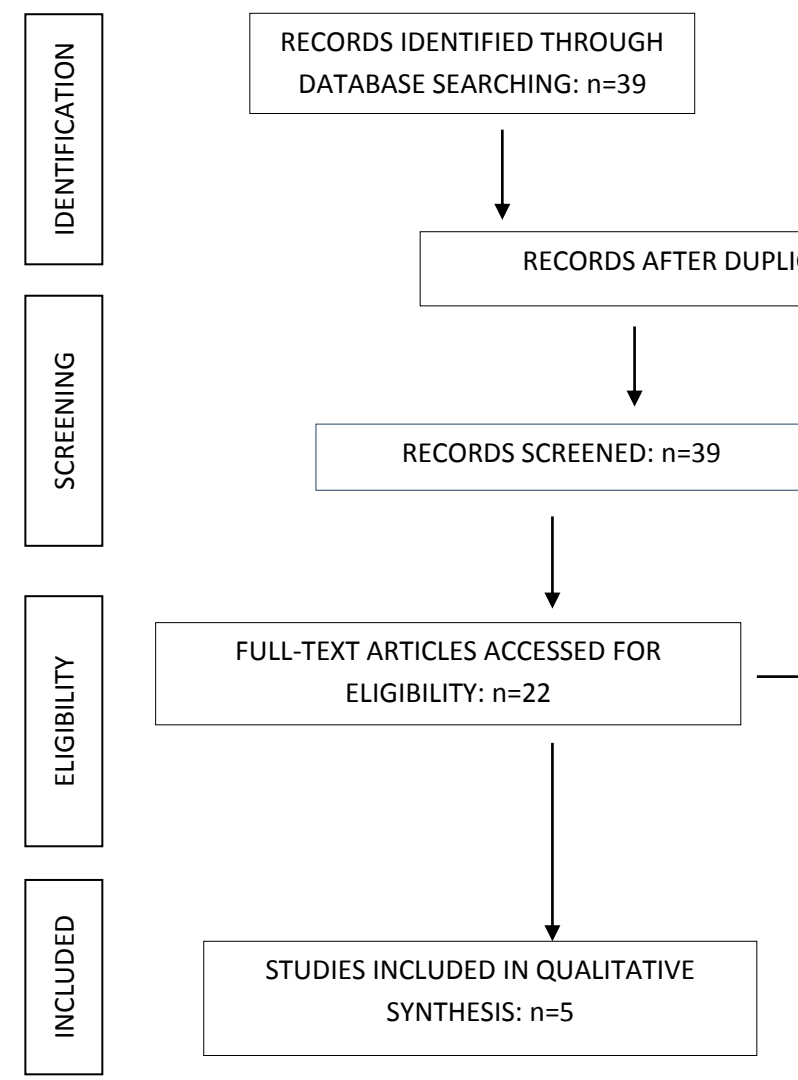

ADDITIONAL RECORDS IDENTIFIED THROUGH

OTHER DATABASE SOURCES: $n=0$

FULL-TEXT ARTICLES EXCLUDED WITH REASONS: $\mathrm{n}=17$

Reasons: $\mathrm{n}=3$ studies using R. rosea interventions with other herbs

$\mathrm{n}=2$ studies assessing mental work

$n=3$ studies assessing endurance exercise performance

$\mathrm{n}=7$ studies assessing the effects of Rhodiola rosea on anaerobic exercise performance, physical and mental

fatigue, stress-related fatigue, skeletal muscle

damage and inflammation, sport performance, skeletal muscle phosphate kinetics, and the effects on oxidants-antioxidants.

$\mathrm{n}=2$ "research in review" of included RCTs (Two pairs of a RCT and a research in review. The research in review $(n=2)$ was excluded)

Figure 2. "Prisma Flowgraph"

major depressive disorder or mild anxiety or mild-moderate depression or participants with life-stress symptoms or healthy participants on stressful activities throughout the trial. The interventions used from the 5 studies were Rhodiola rosea interventions at various doses. The outcomes were assessed with the HAMD questionnaire (Hamilton, 1960), the BDI-II questionnaire (Beck et al., 1996), the MADRS questionnaire (Montgomery, Asberg, 1979), the STAI questionnaire (Spielberger et al., 1983), the Profile of Mood States Inventory (McNair, Lorr, Doppleman, 1971), the SAM test (citation was not found), the SF-36 (Sullivan, Karlsson, Ware, 1995) and the CGI-I (Guy, William, 1976).

\section{Risk of Bias}

A total of five randomized controlled trials were included in our review, all of which were assessed for bias with the Cochrane tool for assessing the risk of bias. Out of the five randomized controlled studies assessed for bias, only one study had a high risk of bias in the domains of allocation concealment and blinding of participants and personnel, while also having an unclear risk of bias in random sequence generation and a low risk of bias in the remaining fields. The rest of the studies had a low risk of bias in all fields (Figure 3). All in all, 4/5 studies included in our review, had a low risk of bias. 


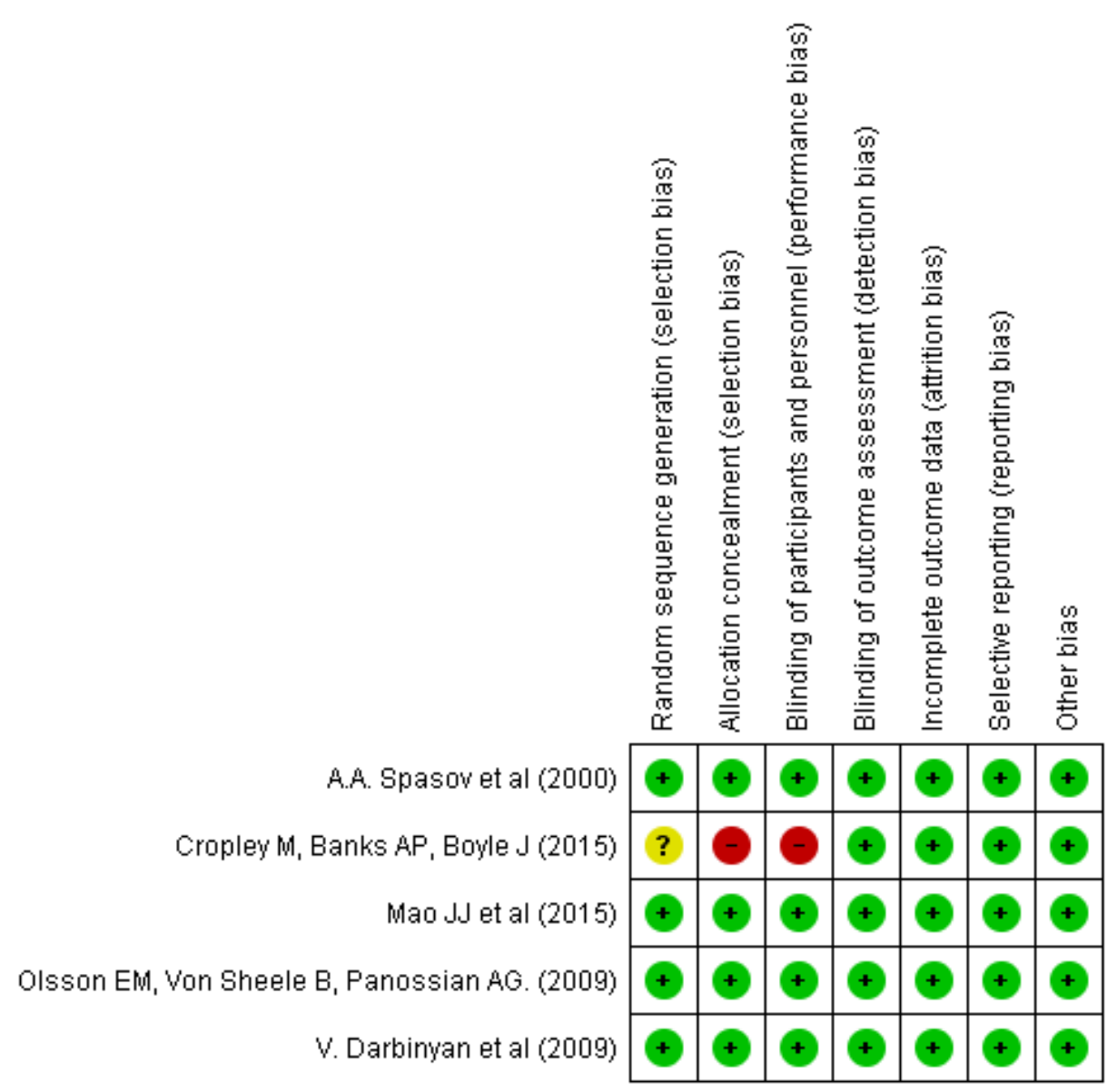

Figure 3. "Risk of Bias Summary"

\section{Findings on depression, anxiety and mood.}

The study of Cropley, Banks and Boyle (2015) demonstrates how participants suffering from mild anxiety responded positively to Rhodiola rosea. Anxiety measured by the STAI significantly decreased for the treatment group in comparison with the placebo group from baseline to endpoint. Total negative mood, anger and confusion measured by the profile of mood states also significantly decreased in the treatment group, in comparison with the placebo group from baseline to endpoint. Depression showed significant main group effects in favour of the treatment group, but significance was not reached when comparing the treatment's group baseline ratings with the endpoint ratings. This occurred because the ratings of the placebo group deteriorated from baseline to endpoint. Thus, while the treatment group did not report significant differences from baseline to endpoint (although improvements were noted), the differences between the treatment and the placebo group at endpoint was significant. The results of Cropley, Banks and Boyle (2015) may indicate that participants suffering from mild anxiety significantly benefited from Rhodiola rosea, while the herb also showed a protective effect against depression in non-depressed participants suffering from anxiety.

The study of Darbinyan et al. 2007 included participants suffering from mild to moderate depression. The treatment groups A and B showed statistically significant improvements from baseline to endpoint in the total HAMD scores in comparison with the placebo group. The BDI score of group A and B also showed statistically significant improvements from baseline to endpoint in comparison with the placebo group. Finally, the HAMD symptom indicator subgroups all improved significantly for group A and B from baseline to endpoint, in comparison with the placebo group (except for the subscale 'symptoms of low self-esteem for group A'). These results may show how Rhodiola rosea can alleviate symptoms of depression in patients suffering from mild to moderate depression.

The study of Mao et al. (2015) included participants suffering from major depression disorder. The Rhodiola rosea 
intervention was compared with sertraline and with placebo. While the HAM-D score decreased in a greater manner for the sertraline group in comparison with the Rhodiola rosea and placebo group, and the HAM-D score decreased in a greater manner for the Rhodiola rosea group in comparison with the placebo group, none of these improvements reached significance. However, there were clinically meaningful odds ratios that indicated that patients taking $R$. rosea had 1.4 times the odds of improvement, and patients on sertraline had 1.9 times the odds of improvement, by week 12 of treatment versus those taking placebo. This study may indicate that while Rhodiola rosea may have antidepressant effects, it is not as efficient as conventional antidepressants for major depression.

The study of Olsson, Von Sheele and Panossian (2009) used a population of fatigued participants. The study reported significant improvements in symptoms of depression and in the quality of life, for both the treatment and the placebo group and no significant difference was found on the comparison. In the same study, the Pines' burnout scale was used to assess fatigue. Statistically significant improvements were found for the treatment group in comparison with the placebo group from baseline to endpoint. This study may demonstrate how the fatigued participants benefited from Rhodiola rosea. The absence of a depression diagnosis on these participants may explain the poor effects of Rhodiola rosea on depression.

Finally, the study of Spasov et al. (2000) included students aged 17-19 years during the course of a stressful examination period. The study reported statistically significant improvements in self-reported mental fatigue $(\mathrm{p}<0.01)$ and statistically significant improvements in general well-being $(\mathrm{p}<0.05)$ in the treatment group, in comparison with the placebo group at endpoint. While speed and accuracy of performance did not improve significantly, the improvement of mental fatigue was more significant than the improvement of general wellbeing. Again, this may be attributed to the mentally stressful examination course.

All in all, we can conclude that Rhodiola rosea's therapeutic effects were in accordance with the medical condition of the participants. Participants suffering from mild anxiety had a reduction in symptoms of anxiety and an improvement of mood. Participants suffering from mild to moderate depression had a decrease in symptoms of depression. Furthermore, participants suffering from major depression saw a decrease in symptoms of depression but not on the same extent as participants treated with sertraline. This may indicate that Rhodiola rosea is less efficient in the treatment of major depression in comparison with conventional antidepressants. Finally, fatigued participants treated with Rhodiola rosea saw improvements in the symptoms of fatigue, but not on depression, while participants embarking on a mentally stressful course reported a decrease in mental fatigue and an increase in general well-being.

\section{DISCUSSION}

Other systematic reviews have also been conducted on the therapeutic effects of the herb. In Pubmed, a total of eight systematic reviews were identified. One review examined the effects of the herb on ischemic heart disease and another examined the effects of the herb on altitude sickness. We believe that these reviews are irrelevant with the outcomes examined in our review. Furthermore, three systematic reviews were identified examining the effects of R. rosea on psychiatric disorders or anxiety or mood (Dwyer, Whitten, Hawrelak, 2011; Sarris et al., 2011; Sarris, 2007). Unfortunately, all three of these reviews included only one study (Darbinyan et al., 2007), which is also included in our review. Thus, no comparisons will be made. Moreover, a systematic review examining the effects of $\mathrm{R}$. rosea on mental and physical fatigue was identified. The review of Ishaque et al. (2012) reports that while some evidence was found for the effects of the intervention on fatigue, this evidence is highly inconclusive due to the low quality of the existing evidence. Furthermore a systematic review examining the effectiveness and efficacy of R. rosea (Hung, Perry, Ernst, 2011) was also identified. This review concludes that beneficial effects were found for physical and mental performance and various mental conditions, some of which were stress-related. It also notes that the herb produced very little adverse effects and that it seems to be relatively safe. A systematic review on numerous herbs for depression was also identified. The review of Yeung et al. (2018) includes four of the five studies included in our review. However, it does not go into great depth, as the primary outcome is not solely the therapeutic effects of Rhodiola rosea but the antidepressant effects of various medicinal herbs. Finally, a non-systematic review was also spotted. The review of Amsterdam and Panossian (2016) provided great information on the mechanism of action of Rhodiola rosea and included both human and animal studies of the herb on depression. Despite the fact that the authors also included Russian literature, this literature was of low quality. No randomized controlled trial that was not included in our review was included in the review of Amsterdam and Panossian (2016).

The chemical composition, the pharmacology and the toxicity of the herb have been examined more rigorously. The studies of Tao et al. (2018), Panossian, Wikman and Sarris (2010) and Amsterdam and Panossian (2016) provide great information about the phytochemistry, the pharmacology, the toxicity and 


\begin{tabular}{|c|c|c|c|}
\hline$\frac{\text { no }}{\text { m }}$ & 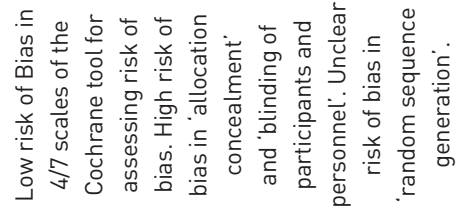 & 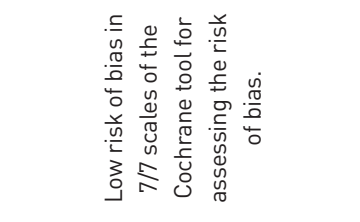 & 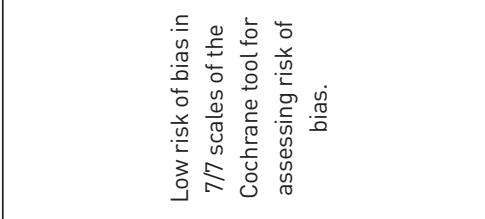 \\
\hline 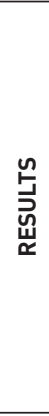 & 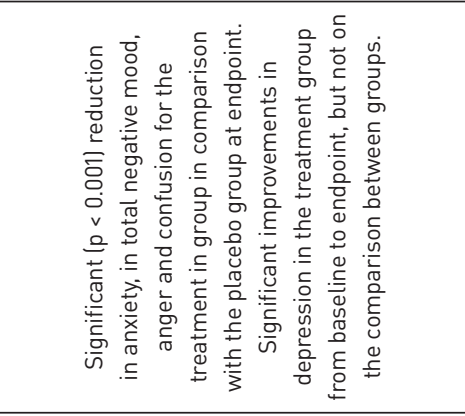 & 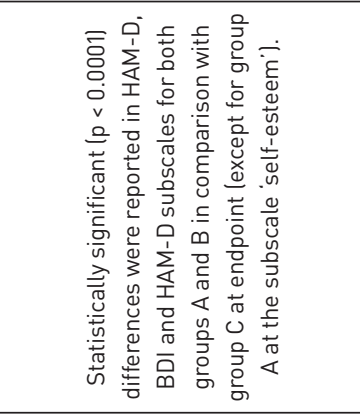 & 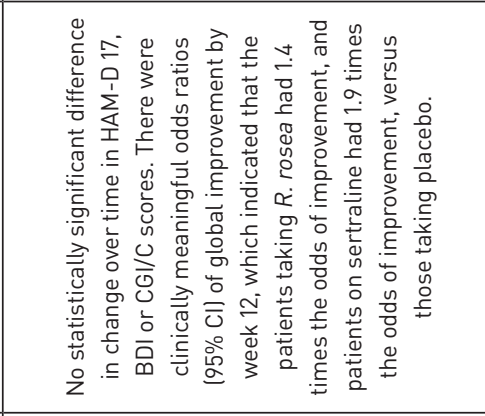 \\
\hline $\begin{array}{l}z \\
\text { 은 } \\
\text { 产 } \\
\text { 它 }\end{array}$ & 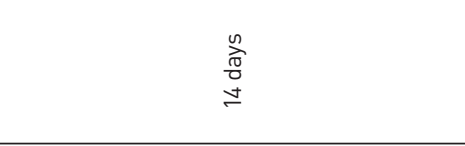 & 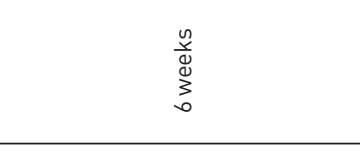 & 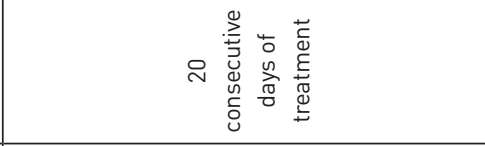 \\
\hline 点 & 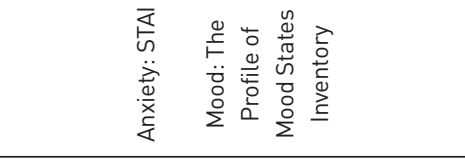 & 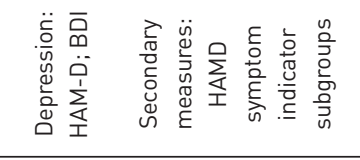 & 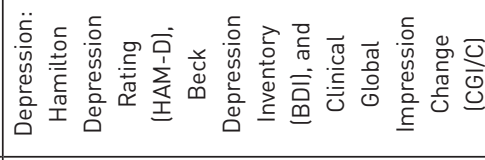 \\
\hline 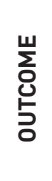 & 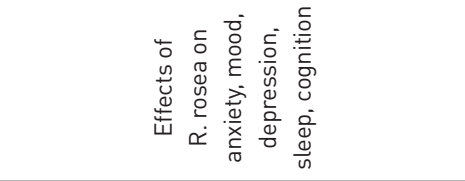 & 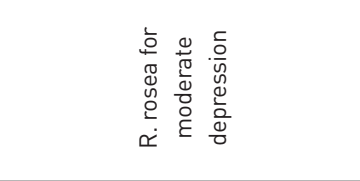 & 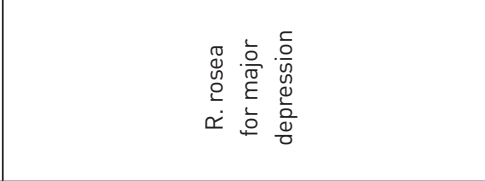 \\
\hline 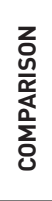 & 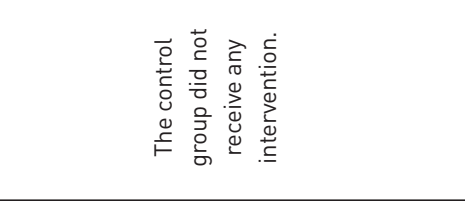 & 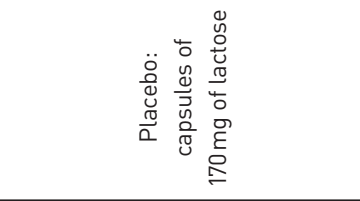 & 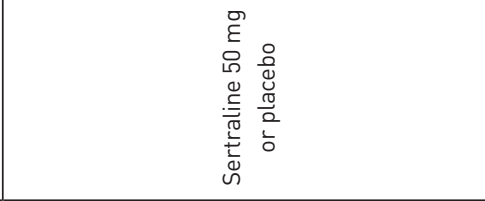 \\
\hline 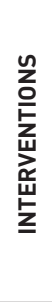 & 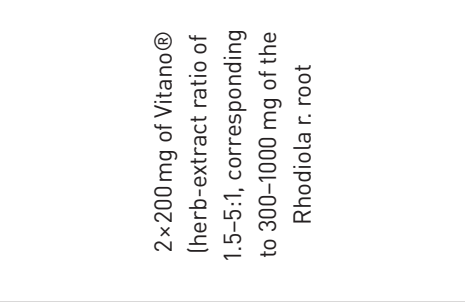 & 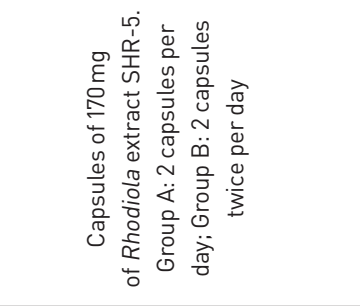 & 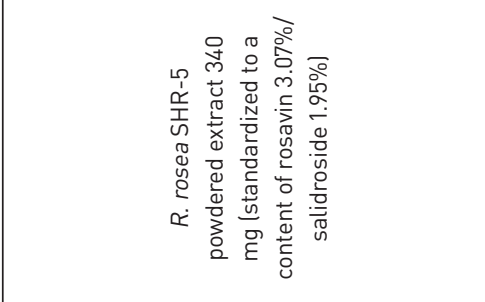 \\
\hline 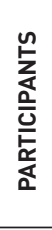 & 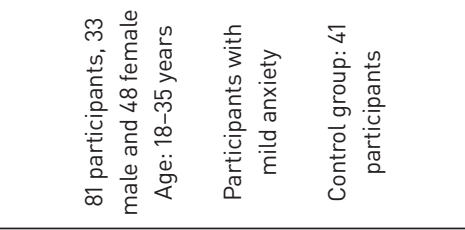 & 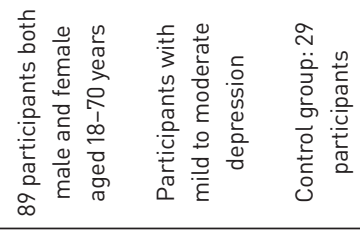 & 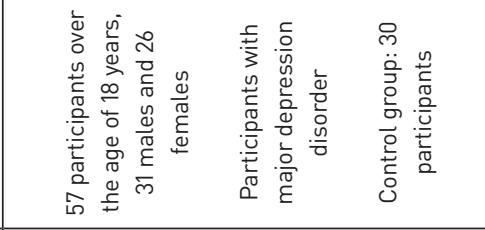 \\
\hline 崖 & 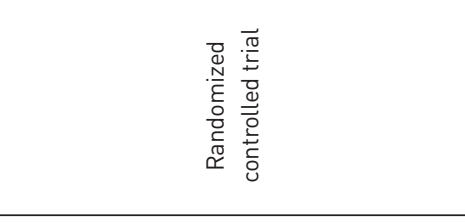 & 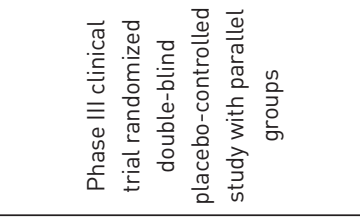 & 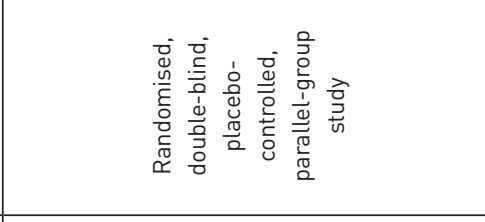 \\
\hline 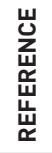 & 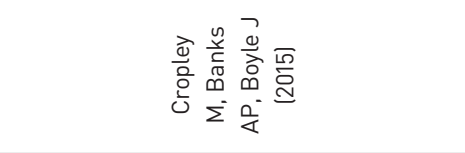 & 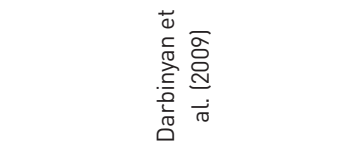 & 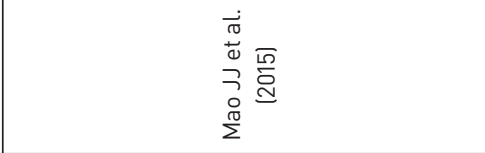 \\
\hline
\end{tabular}




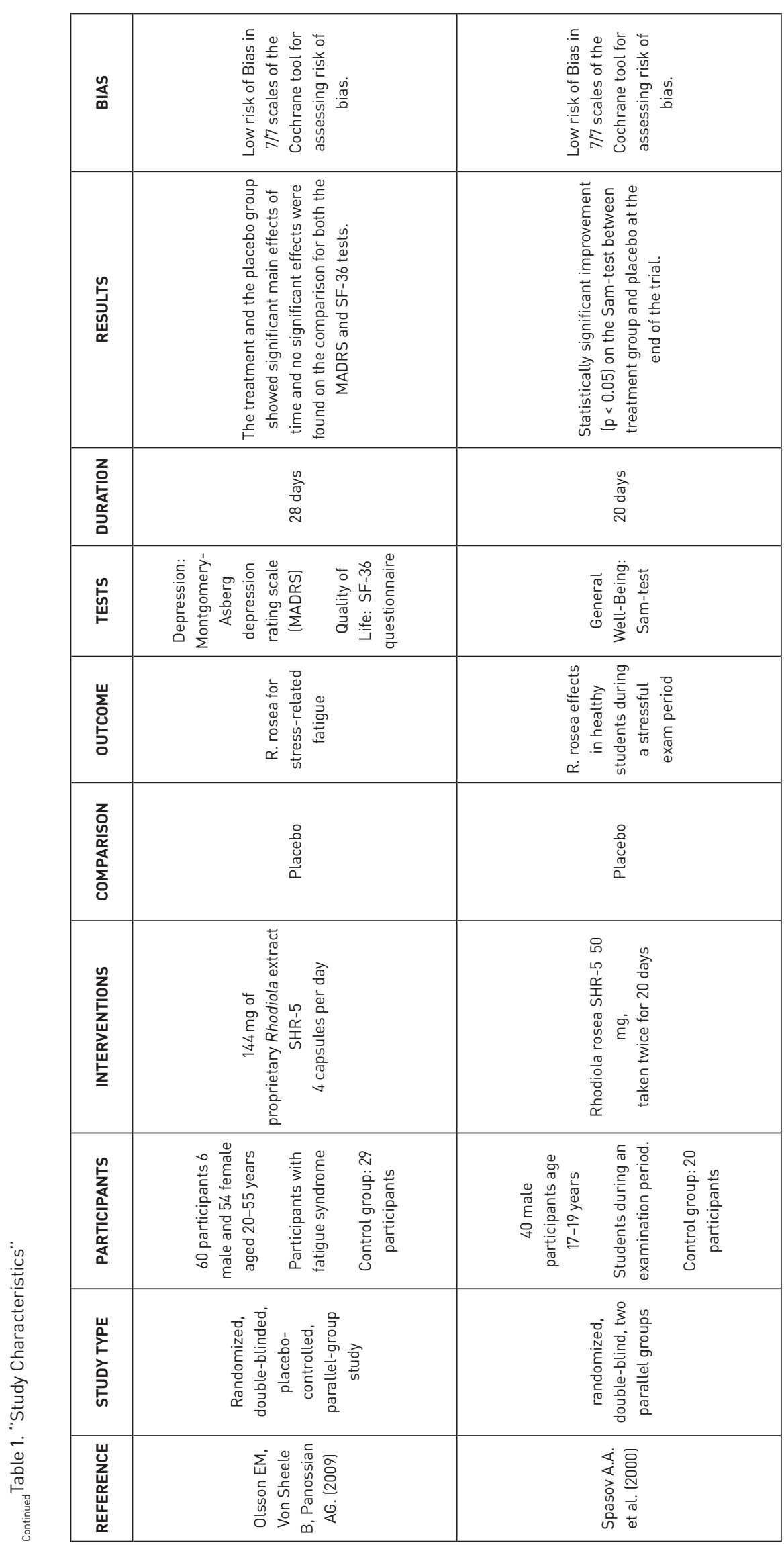


the animal studies performed on the herb. From these studies, it should be highlighted that Rhodiola rosea does not share the same mechanism of conventional antidepressants (Amsterdam, Panossian, 2016). Rhodiola rosea has many different possible therapeutic effects, which include antioxidant, anticancer, antidiabetic, anti-altitude sickness, anti-stress, antidepressant, anxiolytic, neuroprotective, antifatigue, anti-inflammatory, immunomodulatory, for addictive disorders and other effects (Tao et al., 2018). Finally, all three studies suggest that the herb is safe, the adverse effects are rare and mild, high doses do not cause significant adverse effects and that the therapeutic window is large. However, Tao et al. (2018) notes that drug interactions are possible and that the long-term use of the herb may pose some safety risks.

Talking all this into consideration, we conclude that the herb must be further researched. Future research should focus on providing high quality randomized controlled clinical trials with a low risk of bias. Interventions should preferably consist solely of Rhodiola rosea extract and various doses should be tested. Combined interventions of a conventional antidepressant with Rhodiola rosea are also preferable, in order to examine a potential combination therapy. Furthermore, comparisons should be made with both placebo and with conventional antidepressants. The population should preferably consist of participants diagnosed with mild to moderate depression or mild to moderate anxiety and large samples should be used. Finally, the outcomes of depression should be assessed with the HAMD questionnaire (Hamilton, 1960), the BDI-II questionnaire (Beck et al., 1996) and the MADRS questionnaire (Montgomery, Asberg, 1979). The outcomes of anxiety should be assessed with the STAI questionnaire (Spielberger et al., 1983) and the HARS questionnaire (Hamilton, 1959). Mood should be assessed with the Profile of Mood States Inventory (McNair, Lorr, Doppleman, 1971) and the MDMQ dimensions questionnaire (Steyer et al., 1997). Homogeneity of outcome measures and a low risk of bias are essential for the performance of a meta-analysis.

\section{LIMITATIONS}

While a meta-analysis of the results was desirable, the authors decided it would be best not to perform one. This decision was made after observing that only two of the studies included in our review used the same outcome measures. Thus, we gave advice for future research that will enable the performance of a meta-analysis in the discussion column.

\section{CONCLUSION}

Significant results were found for the effects of the herb on mild to moderate depression, mild anxiety and overall mood. Even though these findings are not conclusive, they certainly encourage further research. Future research should aim at providing randomized controlled clinical trials with a low risk of bias and homogeneity of outcome measures. We hope that this systematic review proves to be informative of the therapeutic effects of Rhodiola rosea and encourages further research of the herb.

\section{ACKNOWLEDGEMENTS}

No Acknowledgements

\section{ETHICAL APPROVAL}

As no new data was collected for this study (systematic review), no ethical approval was necessary.

\section{INFORMED CONSENT}

As no new data was collected for this study (systematic review), no informed consent was required.

\section{CONFLICTS OF INTEREST}

The authors declare no conflict of interest in conducting this review.

\section{FUNDING}

This review did not receive any specific grant from funding agencies in the public, commercial, or not-for-profit sectors. 


\section{REFERENCES}

Anghelescu I.G, Edwards D, Seifritz E, Kasper S. (2018) 'Stress management and the role of Rhodiola rosea: a review' International Journal of Psychiatry in Clinical Practice Vol 22 Issue 4 Pages:242-252

Amsterdam JD, Panossian AG. 'Rhodiola rosea L. as a putative botanical antidepressant' Phytomedicine. 2016 Jun 15;23(7):77083. doi: 10.1016/j.phymed.2016.02.009. Epub 2016 Feb 24

Beck AT, Steer RA, Ball R, Ranieri W (December 1996). 'Comparison of Beck Depression Inventories -IA and -II in psychiatric outpatients'. Journal of Personality Assessment. 67 (3): 588 97. doi:10.1207/s15327752jpa6703_13. PMID 8991972

Brekhman I. I.; Dardymov I. V. (1969) 'New substances of Plant Origin which Increase Nonspecific Resistance'. (Annual Review of Pharmacology. 9: 419-430)

Cropley M, Banks AP, Boyle J 'The Effects of Rhodiola rosea L. Extract on Anxiety, Stress, Cognition and Other Mood Symptoms' Phytother Res. 2015 Dec;29(12):1934-9.

Darbinyan V, Aslanyan G, Amroyan E, Gabrielyan E, Malmström C, Panossian A. 'Clinical trial of Rhodiola rosea L. extract SHR-5 in the treatment of mild to moderate depression.' Nord J Psychiatry. 2007;61(5):343-8. Erratum in: Nord J Psychiatry. 2007;61(6):503.

Dwyer AV, Whitten DL, Hawrelak JA 'Herbal medicines, other than St. John's Wort, in the treatment of depression: a systematic review.' Altern Med Rev. 2011 Mar;16(1):40-9. Review.

European Medicines Agency (2008) 'Reflection Paper on the Adaptogenic Concept' Committee on Herbal Medical Products (PDF) 8 May 2008.

Evstavieva L, Todorova M, Antonova D, Staneva J (2010). 'Chemical composition of the essential oils of Rhodiola rosea $L$. of three different origins' Rharmacogn Mag. 6 (24): 256-258.

Guy, William (1976). 'Clinical Global Impressions' ECDEU Assessment Manual for Psychopharmacology-Revised. Rockville, MD: U.S. Department of Health, Education, and Welfare; Public Health Service, Alcohol; Drug Abuse, and Mental Health Administration; National Institute of Mental Health; Psychopharmacology Research Branch; Division of Extramural Research Programs. pp. 218-222.
Hamilton M. (1959) 'The assessment of anxiety states by rating.' $\mathrm{Br}$ J Med Psychol 1959; 32:50-55.

Hamilton, M (1960). 'A rating scale for depression'. Journal of Neurology, Neurosurgery, and Psychiatry. 23: 56-62. doi:10.1136/ jnnp.23.1.56. PMC 495331. PMID 14399272.

Hungk SK, Perry R, Ernst E (2011) 'The effectiveness and efficacy of Rhodiola rosea L.: a systematic review of randomized clinical trials.' Phytomedicine. 2011 Feb 15;18(4):235-44. doi: 10.1016/j. phymed.2010.08.014. Epub 2010 Oct 30.

Ishaque S, Shamseer L, Bukutu C, Vohra S (2012) 'Rhodiola rosea for physical and mental fatigue: a systematic review.' BMC Complement Altern Med. 2012 May 29;12:70. doi: 10.1186/14726882-12-70.

Jacob Cohen (1988) 'Statistical Power Analysis for the Behavioral Sciences. (2nd ed.), New Jersey: Lawrence Erlbaum Associates.

Lishmanov I, Trifonova Z, Tsibin AN, Maslova LV, Dement'eva LA (1987) '[Plasma beta-endorphin and stress hormones in stress and adaptation]'. Biull Eksp Biol Med. 1987 Apr;103(4):422-4.

Mao JJ, Xie SX, Zee J, Soeller I, Li QS, Rockwell K, Amsterdam JD 'Rhodiola rosea versus sertraline for major depressive disorder: A randomized placebo-controlled trial' Phytomedicine. $2015 \mathrm{Mar}$ 15;22(3):394-9.

Mao Y, Li Y, Yao N (2007) 'Simultaneous determination of salidroside and tyrosol in extracts of Rhodiola L. By microwave assisted extraction and high-performance liquid chromatography'. Journal of Pharmaceutical and Biomedical Analysis.45 (3): 510-5.

Mattioli L, Funari C, Perfumi M (2008) 'Effects of Rhodiola rosea $\mathrm{L}$. extract on behavioural and physiological alterations induced by chronic mild stress in female rats.' Journal of Psychopharmacology. 2008 May 30.

McNair, D.; Lorr, M.; Doppleman, L. (1971). POMS Manual for the Profile of Mood States. San Diego, CA: Educational and Industrial Testing Service.

Moher D, Liberati A, Tetzlaff J, Altman DG The Prisma Group (2009) 'Preferred Reporting Items for Systematic Reviews and Meta-Analyses: The PRISMA Statement.' Journal of Clinical Epidemiology 2009 
Moher D, Liberati A, Tetzlaff J, Altman DG.

Montgomery SA, Asberg M (April 1979). 'A new depression scale designed to be sensitive to change'. British Journal of Psychiatry. 134 (4): 382-89. doi:10.1192/bjp.134.4.382. PMID 444788.

Olsson EM, Von Sheele B, Panossian AG (2009) A Randomised, Double-Blind, Placebo-Controlled, Parallel-Group Study of the Standardised Extract SHR-5 of the Roots of Rhodiola rosea in the Treatment of Subjects with Stress-Related Fatigue'. Planta Med. 2009 Feb;75(2):105-12.

Panossian A, Wikman G, Hambardzumyan M, Hovhanissyan A 2007 'The Adaptogens Rhodiola and Schizandra Modify the Response to Immobilization Stress in Rabbits by Suppressing the Increase of Phosphorylated Stress-activated Protein Kinase, Nitric Oxide and Cortisol’ Drug Target Insights 2007; 2: 39-54.

Panossian A, Wikman G, Sarris J (2010). 'Rosenroot (Rhodiola rosea): Traditional use, Chemical composistion, pharmacology and clinical efficacy'. Phytomedicine. 2010 Jun;17(7):481-93.

Panossian A, Wikman G, (2010) 'Effects of Adaptogens on the Central Nervous System and the Molecular Mechanisms Associated with Their Stress-Protective Activity Pharmaceuticals (Basel).' 2010 Jan; 3(1): 188-224.

Perfumi M, Mattioli L (2007) Adaptogenic and central nervous system effects of single doses of $3 \%$ rosavin and $1 \%$ salidroside Rhodiola rosea L. extract in mice.' Phytotherapy Research. 2007 Jan;21(1):37-43.

Plants for a Future 2012 https://pfaf.org/user/Plant. aspx? LatinName=Rhodiola+rosea

Ross SM 'Rhodiola rosea (SHR-5), Part 2: A Standardized Extract of Rhodiola rosea Is Shown to Be Effective in the Treatment of Mild to Moderate Depression' Holist Nurs Pract. 2014 MayJun;28(3):217-21.

Sarris J 'Herbal medicines in the treatment of psychiatric disorders: a systematic review.' Phytother Res. 2007 Aug;21(8):703-16. Review.

Sarris J, Panossian A, Schwitzer I, Stough C, Scholey A 'Herbal medicine for depression, anxiety and insomnia: a review of psychopharmacology and clinical evidence.' Eur Neuropsychopharmacol. 2011 Dec;21(12):841-60. doi: 10.1016/j. euroneuro.2011.04.002. Epub 2011 May 23. Review.
Spasov A.A., Wikman G.K., Mandrikov V.B., Mironova A., Neumoin V.V.NEUMOIN (2000) A double-blind, placebo-controlled pilot study of the stimulating and adaptogenic effect of Rhodio/a rosea SHR-5 extract on the fatigue of students caused by stress during an examination period with a repeated low-dose regimen.' Phytomedicine, Vol. 7(2), pp. 85-89.

Spielberger, C. D., Gorsuch, R. L., Lushene, R., Vagg, P. R., \& Jacobs, G. A. (1983). Manual for the State-Trait Anxiety Inventory. Palo Alto, CA: Consulting Psychologists Press.

Steyer R., Schwenkmezger P., Notz P., \& Eid M. (1997). The multidimensional mental state questionnaire: Manual. Gottingen: Hogrefe.

Sullivan M, Karlsson J, Ware J. The Swedish SF-36 health survey - I. Evaluation of data quality, scaling assumptions, reliability and construct validity across general populations in Sweden. Soc Sci Med. 1995; 41 1349-58

Tao H, Wu X, Cao J, Peng Y, Wang A, Pei J, Xiao J, Wang S, Wang $Y$. 'Rhodiola species: A comprehensive review of traditional use, phytochemistry, pharmacology, toxicity, and clinical study.' Med Res Rev. 2019 Sep;39(5):1779-1850. doi: 10.1002/med.21564. Epub 2019 Jan 16.

Van Diermen D, Maston A, Bravo J, Reist M, Carrupt PA, Hostettmann K (2009) 'Monoamine oxidase inhibition by Rhodiola rosea L. roots.' J Ethnopharmacol. 2009 Mar 18;122(2):397-401. doi: 10.1016/j.jep.2009.01.007. Epub 2009 Jan 9.

Yang S.J., Yu H.Y., Kang D.Y., Ma Z.Q., Qu R., Fu Q., Ma S.P.Antidepressant-like effects of salidroside on olfactory bulbectomy-induced pro-inflammatory cytokine production and hyperactivity of HPA axis in rats. Pharmacol. Biochem. Behav, 124 (2014), pp. 451-457.

Yeung KS, Hernandez M, Mao JJ, Haviland I, Gubili J. 'Herbal Medicine for Depression and Anxiety: A Systematic Review with Assessment of Potential Psycho-Oncologic Relevance'. Phytother Res. 2018 May;32(5):865-891. doi: 10.1002/ptr.6033. Epub 2018 Feb 21. Review. 